\title{
ERFAHRUNGEN Mit SIMULIERTEN SELEKTIONSEXPERIMENTEN IN DER LEHRE
}

B. BRuns und P. GlodeK. - Institut für Tierzucht und Haustievgenetik, Universilät Göttingen

Es wurde ein simuliertes Selektionsexperiment als Hilfsmittel in der Haustiergenetiklehre eingesetzt. Der Simulation lag ein statistisch-biologisches Modell zugrunde zur Erstellung von zwei Selektionsmerkmalen, der Wurfgröße und dem Ferkelgewicht.

Die gesammelten Erfahrungen lassen sich dahingehend zusammenfassen:

1. Die Simulation von zwei Selektionskriterien eröffnet vielfältigere Möglichkeiten zur Demonstration genetischer Zusammenhänge als die Simulation von nur einem Merkmal.

2. Die Selektionskriterien sollten für jedes simulierte Individuum vorhanden sein zur möglischt exakten Schätzung der Generationsmittel. Die Wurfgröße als Selektionsmerkmal ist weniger gut geeignet. Eine Population sollte mindestens roo Tiere umfassen.

3. Die statistisch-biologischen Modelle sollten möglichst einfach sein.

4. Die Zahl zu vergleichender Selektionsverfahren sollte gering gehalten worden, aber jedes Verfahren sollte mehrmals wiederholt werden.

5. Simulationsexperimente sind nützliche Hilfsmittel zur Gestaltung von praktischen Kursen in der Tierzüchtung und zur Ergänzung der Grundlagenvorlesungen.

\section{TEACHING BREEDING PLANNING FOR A DUAL-PURPOSE CATTLE POPULATION BY A PROGRAMMED EXERCISE}

\section{P. H. Petersen. - Institute of Animal Science, The Royal Veterinary and Agricultural University $D K-1958$ Copenhagen $V$, Denmark}

A programmed exercise has been developed in order to give a comprehensive and activating teaching of the principles in breeding planning within a dual-purpose cattle population. In the initial round groups of students gradually build up their breeding plans within a given selection scheme and calculate the expected genetic and economic responses. In the following rounds the input factors are changed one at tha time in order to improve the results, and the expected results are calculated by a computer program. The experience from 7 rounds carried through of 6 groups is discussed.

Contribution A L'ENSEIGNEMENT DE LA GÉNÉtiQUE :

PARTITION DU GÉNOTYPE ET FORMULES DE PRÉDICTION EN GÉNÉTIQUE QUANTITATIVE

R. Hanset. - Fasulté de Médecine Vétévinaire, rojo Bruxelles, Belgique

L'établissement des formules classiques de corrélation ou de régression entre génotypes et phénotypes est réalisé, avec comme point de départ : I) la partition du génotype en une partie commune aux individus d'une même famille et une partie aléatoire propre à chaque génotype; 2) la variance d'une moyenne de données en corrélation. En guise d'applications, on établit la formule de : I) la corrélation entre frères de même portée; 2) la covariance entre filles futures et filles actuelles d'un taureau; 3) la corrélation entre un caractère de la mère et un autre caractère de la fille; 4) la régression des filles futures sur les filles actuelles; 5) la régression du génotype d'un verrat sur la moyenne de ses $m n$ descendants, $m$ portées de $n$ porcelets; 6 ) la régression du génotype d'un individu sur la moyenne de $n$ frères, l'individu concerné étant inclus dans la moyenne; 7) la covariance phénotypique entre un caractère $X$ mesuré sur $n_{1}$ frères et un caractère $Y$ mesuré sur $n_{2}$ frères, $\mathrm{N}$ frères étant communs aux deux groupes.

\section{Prühungen in Tierzucht für Studenten}

\section{F. WeBer. - Institut für Tierproduktion, Gruppe Tierzucht Eidgen. Technische Hochschule} Zürich.

In einer Prüfung sollen die Kandidaten darauf hin beurteilt werden, ob sie ihr Wissen anwenden können und in der Lage sind, begründete Zuchtentscheide zu treffen. Schriftliche Prüfungen sind den mündlichen vorzuziehen, und die Kandidaten sollen dabei alle Unterlagen benützen 
können. Anstelle von Definitionsfragen sind den Kandidaten Situationen und Fälle vorzulegen, die sie zu beurteilen haben. Die Fragen sollten nicht lauten : "Was verstehen Sie unter...?", sondərn "Was würden Sie tun, wenn...? ", "Was halten Sie davon?", "Wieviel ergibt das? ".

Die Fähigkeit, Wissen anzuwenden und Entscheide zu treffen muss allerdings auch ausserhalb der Prüfung in allen Unterrichtsveranstaltungen systematisch gefördert werden.

\section{V. - Communications libres}

Phenotypic AND Genetic PaRAMeters of the activities oF NADPH-GENERATING ENZYMEs IN SWINE ADIPOSE TISSUE

C. Strutz and E. Rogdakis. - Departement of Animal Breeding at the Institute of Animal Husbandry and Breeding Hohenheim University, Germany.

Preliminary results are reported out of a German Landrace half-sibs population analysis for estimation of phenotypic parameters as well as heritabilities and genetic correlations of lipogenic enzyme activities in porcine adipose tissue. For determination of the NADPH-generating enzymes NADP-malate dehydrogenase (NADP-MDH), NADP-isocitrate dehydrogenase (NADPICDH), glucose-6-phosphate-(G-6PDH) and 6-phosphogluconate dehydrogenase (6-PGDH) subcutaneous adipose tissue samples were taken by biopsy at the growth stages of $4^{\circ}$ and roo kg live weight. For the first measurement 692 gilts from 230 sires and for the second measurement 62 I gilts from 227 sires were examined. The heritabilities of the enzyme activities range due to the reference units used - from moderate (19\%) to very high values $(72 \%)$. The genetic and phenotypic correlations to the degree of carcase fatness are highly significant at measurement 2. Though adipose tissue cellularity is assumed to influence the extent of estimated genetic parameters the use of lipcgenic enzyme activities as selection criteria seems to be justified.

\section{Modellversuche zur Selektion auf Adaptation un Wachstum}

J. H. Weniger, P. Horst, D. Steinhauf, E. S. Tawfik, F. MAjor. - Institut für Tierproduktion der technischen Universität Berlin, B.R.D.

Die Selektion von Mäusen auf die Lerkmale Belastbarkeit (Uberlebenszeit unter starker physischer Belastung) und Proteinansatz bis zum Alter von 6o Tagen, wobei in positiver und negativer Richtung jeweils in zwei analogen Linien selektiert wurde, erbrachte bis zum Ablauf der zwölften Selektionsgeneration folgende Ergebnisse : Zwischen den in positiver und negativer Richtung selektierten Linien zeigt sich im Falle der Seleksion auf Proteinmenge von der zweiten Selektionsgeneration an eine hochgesicheste Differenz. Bei der Selekticn auf Belastberkeit ist dies von der sechsten Selektionsgeneration an der Fall. Werden die beiden analogen Linien zusammengefaßt, zeigt sich die statistisch hochsignifikante Differenzierung zwischen den Selektionsrichtungen auch hier schon in der zweiten Selektionsgeneration. In beiden Fällen ist offensichtlich der realisierte Erfolg bei der Selektion in positiver Richtung stärker als bei der in negativer Richtung. Der Befund, daß bei steigender Belastbarkeit, gemessen an der Uberlebenszeit unter starker Belastung, die Varianz unverhältnismässig stark ansteigt, bedarf noch weiterer Prüfung, um einer Interpretation zugänglich zu sein.

Unter dem Einfluß der Selektion auf Belastbarkeit ändert sich das Körpergewicht der Tiere insofern, als belastungsfähigere Tiere leichter und belastungsanfälligere Tiere schwerer werden. Die analoge Tendenz bei der Selektion auf Proteinansatz ist jedoch weniger scharf ausgeprägt. Die Vermutung, daß zwischen Belastbarkeit und Proteinansatz bzw. Körpergewicht eine negative Beziehung besteht, konnte bis $z u$ einem gewissen Grade durch die Korrelation zwischen dem Gewicht der Tiere am 6o. Tag und der Uberlebenszeit in Höhe von $\gamma=-0,22^{+++}$bestätigt werden.

\section{Enzymaktivitäten im Blutserum von Mäusen unter dem Einfluss Der Selektion auf Belastbarkeit}

F. Major, E. S. TAwhi K, D. SteInhauf. - Institut für Tierproduction der Techhischen Universität Berlin, B.R.D.

An 59r Mäusen, die aus Selektionslinien stammten, welche auf hohe und niedere Belastbarkeit unter extremen Umweltbedingungen selektiert waren, wurden verschiedene Enzymaktivitäten im Blutserum getestet. Folgende Ergebnisse konnten ermittelt werden : 